\section{Anaesthesia for neonatal surgical emergencies}

Richard J. Palahniuk MD

\begin{abstract}
Administering a safe anaesthetic to a sick neonate is dependent to the greatest extent on the skill and experience of the anaesthetist and to a much lesser extent on the techniques and agents selected. For this reason, this refresher course will focus on the physiological, pharmacological and medical challenges the neonate presents rather than present a "cookbook" approach to the anaesthetic management of a selected series of surgical procedures.
\end{abstract}

\section{Neonatal physiology}

Unfortunately, the word neonate is applicable to a wide spectrum of babies under 44 weeks gestational age. The 30-week, 1000-gram infant undergoing ligation of a patent ductus is a substantially different challenge from the three-week-old full term baby undergoing pyloromyotomy. As a result, it may be slightly inaccurate to generalize when discussing "neonatal" anaesthesia. Such inaccuracies will be indicated wherever possible.

\section{Respiratory system}

The neonate has a high respiratory rate, a low FRC, a high closing capacity and a high oxygen consumption (6 $\mathrm{ml} \cdot \mathrm{kg}^{-1} \cdot \mathrm{min}^{-1}$ vs 3 in the adult). This combination of factors makes him susceptible to the rapid development of hypoxaemia. Smooth, rapid intubation procedures and carefully planned extubations are much more critical in neonates than other patients. Since neonatal intubations can be difficult (large tongue, large epiglottis, cephalad glottis), one cannot overemphasize the need for careful preparation and optimal conditions for the neonatal intubation. Another point to remember is that placing a tracheal tube reduces the neonatal airway diameter by as much as 25 per cent and increases airway resistance to laminar gas flow by three times. This problem can be even worse when using smaller tracheal tubes in premature neonates. Spontaneous ventilation may thus be associated with very high work of breathing in intubated neonates.

With immature diaphragmatic development and underdeveloped intercostal muscles, neonates have less capacity to increase ventilation and are more susceptible to ventilatory depressants. Especially in the immediate postoperative period, hypothermia, residual drug effects and increased ventilatory demands may result in respiratory failure.

\section{Cardiovascular system}

Neonates depend on heart rate to maintain cardiac output and adequate tissue perfusion. This results from their poorly compliant myocardium. Any stimulus which produces bradycardia may have deleterious effects on the neonate. Also, neonates (especially prematures) have fragile baroresponse mechanisms ${ }^{\prime}$ which may aggravate the cardiovascular depression produced by various stimuli and cardiac depressant drugs such as halothane.

Neonates also respond to a number of stimuli (cold, pain) by increasing pulmonary vascular resistance more than systemic vascular resistance. This may result in right to left shunting across the previously closed foramen ovale and ductus arteriosus.

\section{Renal function}

Neonates have a low glomerular filtration rate and a poor capacity to conserve water and concentrate their urine. These may have significance in the excretion of drugs and drug metabolites and in their renal responses to dehydration.

\section{Thermoregulation}

Neonates are prone to hypothermia. Their large surface area in relation to body weight, their relative lack of subcutaneous fat (especially premature infants), and their inability to generate heat by normal mechanisms are responsible for this problem. Hypothermia can result in increased oxygen consumption, respiratory depression, and delayed recovery from depressant medications. For these reasons, the anaesthetist must pay meticulous attention to maintaining body temperature during neonatal anaesthetics. Keeping the operating room uncomfortably warm, heating and humidifying inspired gases, warming IV and prep solutions, keeping the baby's body

Department of Anaesthesia, University of Manitoba, Winnipeg, Manitoba. 
and head insulated, and using radiant heaters are a few of the means available to keep the neonate warm.

\section{Glucose}

Neonates are at risk of developing hypoglycaemia because of low glycogen stores and reduced capacity for gluconeogenesis. This risk is increased in premature infants, small for gestational age babies, babies of diabetic mothers and septic infants. Monitoring of plasma glucose levels and maintaining adequate glucose infusions are important considerations during neonatal anaesthetics.

\section{Miscellaneous}

Other physiological characteristics of the neonate which may have an influence on the anaesthetic and perioperative management include:

1 Immature liver enzyme systems

2 Increased plasma bilirubin

3 Tendency to hypocalcaemia

4 Low plasma albumin

5 Low plasma cholinesterase

6 Risk of retrolental fibroplasia

7 High blood volume

8 High extracellular fluid volume

\section{Pharmacology of drugs used in the neonate ${ }^{2}$}

\section{Pharmacokinetics}

Neonates generally have a high initial volume of distribution of drugs administered IV as well as a prolonged elimination half-life of many agents. Plasma protein binding of some drugs is reduced when compared with that in older children and adults.

\section{Uptake and distribution of inhaled agents}

Because of their relatively high alveolar ventilation and low functional residual capacity, neonates achieve higher alveolar concentrations of inhaled anaesthetics more quickly than older patients. This can result in early profound depression of cardiovascular and ventilatory systems.

\section{Anaesthetic requirements}

There is no doubt that neonates feel pain and exhibit stress responses to surgical and other stimuli. ${ }^{3}$ Except when moribund, they require anaesthetics for surgical procedures. Neonates can mount substantial hormonal and metabolic stress responses to surgery which can be attenuated by adequate levels of anaesthesia. ${ }^{4}$ In addition, laryngoscopy and intubation are associated with increases in intracranial pressure in the awake neonate which can be attenuated by prior anaesthesia. ${ }^{5}$

The anaesthetic requirement for inhaled agents (MAC) appears to be substantially less in term neonates than in older infants ${ }^{6}$ and even less in preterm babies. ${ }^{7}$ There is also the clinical impression that the depressant effect of IV anaesthetics is greater in neonates than older infants.

\section{Nitrous oxide}

Although useful in the neonate, nitrous oxide is contraindicated in many neonatal surgical emergencies (congenital diphragmatic hernia, gastroschisis, necrotizing enterocolitis, bowel obstruction). In these infants, air-oxygen mixtures should be used to achieve the appropriate $\mathrm{FiO}_{2}$.

\section{Volatile anaesthetics}

Halothane and isoflurane are both useful as induction and maintenance agents in the neonate. Previous comments about the rapid elevation of alveolar partial pressure, rapid development of cardiorespiratory depression and reduced MAC values should be remembered.

\section{Narcotics}

Although fentanyl is becoming more popular in neonatal anaesthesia, ${ }^{8}$ dose requirements are probably lower than in older children and prolonged respiratory depression may be a problem. Higher doses should only be used when at least 24 hours of postoperative ventilatory support is planned. Any narcotic used to supplement the anaesthetic technique or for analgesia can be expected to produce more profound and long-lasting respiratory depression in the neonate than in other patients.

\section{Succinylcholine}

Higher doses of succinylcholine ( $2 \mathrm{mg} \cdot \mathrm{kg}^{-1}$ ) are required to produce adequate relaxation in the neonate. Since neonates do not fasciculate or get elevations in intragastric pressure from succinylcholine, precurarization is not required. Neonates do, however, frequently become bradycardic following succinylcholine, and routine atropine premedication is recommended $\left(0.02 \mathrm{mg} / \mathrm{kg}^{-1} \mathrm{IM}\right.$ or $0.01 \mathrm{mg} \cdot \mathrm{kg}^{-1} \mathrm{IV}$ ).

\section{Non-depolarizing muscle relaxanis}

Neonates demonstrate reduced neuromuscular function when compared with older children even without the administration of muscle relaxants. Dosages of the non-depolarizing relaxants may be less than recommended for adults ${ }^{9}$ and careful monitoring of neuromuscular function can be a useful adjunct to neonatal anaesthesia. Atracurium is becoming popular for neonates because of its short duration of action and its unique disposal in the body. Pancuronium is still useful where prolonged block is desirable and postoperative ventilatory support is planned.

Reversal of neuromuscular blockade can be satisfacto- 
rily achieved by edrophonium, $1 \mathrm{mg} \cdot \mathrm{kg}^{-1}$ preceded by atropine, $0.015 \mathrm{mg} \cdot \mathrm{kg}^{-1}$.

\section{Local anaesthetics}

Regional anaesthesia to supplement the general anaesthetic and/or to provide postoperative analgesia is becoming increasingly popular. ${ }^{10}$ Bupivacaine to a total dose of 2.0 $\mathrm{mg} \cdot \mathrm{kg}^{-1}$ can be used for local infiltration, nerve blocks or caudal blocks.

\section{Monitoring}

Although the size of the neonate can make certain types of monitoring technically difficult, this should not deter the anaesthetist from instituting the appropriate required monitoring in each baby. The minimum required monitoring should include:

1 Continuous EKG

2 Continuous or intermittent BP

3 Precordial/oesophageal stethoscope

$4 \mathrm{FIO}_{2}$

5 Pulse oximetry ${ }^{11}$

6 Continuous core temperature

Additional monitoring may be indicated in some situations:

1 Inspired airway pressure

2 Urine output

3 Central venous pressure

4 Neuromuscular function

5 Intermittent arterial blood gases

and acid-base balance

6 Blood glucose

Temperature monitoring, as a vital component of the monitoring systems, deserves special comment. Rectal temperature may only slowly reflect changes in core temperature depending on the contents of the rectum. Similarly, oesophageal temperature may reflect airway temperature more than core body temperature especially when used at the distal end of an oesophageal stethoscope. Nasopharyngeal or lower oesophageal temperature may more accurately reflect core temperature and changes in body temperature.

\section{Specific surgical emergencies}

I will not discuss these exhaustively either from the surgical or anaesthetic view point. Specific challenges as they apply to the anaesthetist will be highlighted. The anaesthetist should remember that neonates who have one abnormality frequently have other congenital abnormalities. Careful preoperative evaluation of neonates is mandatory not only to assess the airway, state of hydration, and respiratory status, but also to be sure there is not a previously undetected congenital heart lesion which could compromise the anaesthetic management.
Hypertrophic pyloric stenosis ${ }^{2}$

Neonates with this disorder are among the least challenging of the neonatal surgical emergencies. It has often been stated that this is a medical rather than a surgical emergency and I can only re-emphasize that. Adequate preoperative attention to rehydration, correction of electrolyte abnormalities and gastric suctioning should ensure a relatively healthy neonate presenting for anaesthesia.

The major challenge to the anaesthetist is securing the airway. After gastric suctioning, a rapid sequence induction with cricoid pressure is the optimal method of assuring adequate conditions for intubation. Some anaesthetists recommend awake intubation, but with proper medical preparation, these babies should be vigorous and not inclined to allow easy access to their glottis.

Following the surgery, these neonates should be extubated when fully awake and observed carefully for the first few minutes after extubation.

\section{Congenital diaphragmatic hernia}

The earlier in the neonatal period the neonate with this disorder demonstrates respiratory distress, the more difficult the management and the more unsatisfactory the outcome. Awake intubation is indicated with careful respiratory control and monitored inspiratory pressures. Excessive airway pressures can result in a pneumothorax on the non-operated side $^{13}$ with obvious disastrous results. The anaesthetist should be prepared for major fluid shifts with large volumes of IV fluids required to maintain venous return and blood pressure. Congenital diaphragmatic hernias are associated with congenital heart defects in 25 per cent of cases.

\section{Patent ductus arteriosus ${ }^{8}$}

Ligation of a PDA is usually indicated in premature neonates after failure of medical management of persistent fetal circulation. Because of the (lack of) size of these babies, technical difficulties in monitoring, temperature control and fluid management can be almost overwhelming. The surgical procedure should be short, but lung retraction can compromise oxygenation and cardiac output. Careful monitoring, including direct arterial blood pressure, is mandatory. Hyperoxia should be avoided because of the risk of producing retrolental fibroplasia.

\section{Necrotizing enterocolitis}

This also is a disorder of premature neonates. In addition to all the usual challenges of anaesthetizing the premature infant, this group of babies presents the additional problem of major fluid shifts. Relatively massive amounts of fluids may have to be administered without clear guidelines to follow. Careful monitoring of blood pres- 
sure (arterial line), heart rate, urine output and acid-base balance are the main determinants of fluid replacement.

\section{Meningomyelocoele}

Neonates with this disorder may require several operative procedures in the first few weeks of life. The first procedure, to cover the defect in the lumbosacral region may be the most challenging for the anaesthetist. A difficult tracheal intubation can be made more difficult by the strange positioning required in these babies.

\section{Tracheoesophageal fistula}

Although tracheoesophageal fistula is most often diagnosed early in the neonatal period, the baby may present a problem to the anaesthetist because of aspiration pneumonitis and/or dehydration. Airway management requires that the tracheal tube be positioned very precisely - in the trachea but below the fistula. This can be achieved by intubating one or other main stem bronchus and then withdrawing the tube until it is just above the carina and bilateral breath sounds are heard. Positive pressure ventilation should be avoided, especially prior to proper placement of the tracheal tube, to prevent insufflation of the stomach. Care should be taken to rule out congenital heart disease which is present in about 25 per cent of these neonates.

The baby should be observed carefully following extubation to ensure that tracheomalacia is not present as well, resulting in partial airway obstruction.

\section{Gastroschisis $^{14}$}

Gastroschisis is associated with major fluid shifts requiring careful attention to fluid and electrolyte management. Primary closure of the abdominal wall may result in elevated intra-abdominal pressure with compromised renal perfusion and/or impaired venous return.

\section{References}

1 Gregory $G A$. The baroresponses of preterm infants during halothane anaesthesia. Can Anaesth Soc J 1982, 29: 105-7.

2 Cook $D R$. Newborn anaesthesia: pharmacologic considerations. Can Anaesth Soc J 1986; 33: S38-S42.

3 Anand KJS, Aynsley-Green A. Measuring the severity of surgical stress in newborn infants. J Ped Surg 1988; 23: 297-305.

4 Anand KJS, Sippell WG, Schofield NM, Aynsley-Green $A$. Does halothane anaesthesia decrease the metabolic and endocrine stress responses of newborn infants undergoing operation? Br Med J 1988; 296: 668-72.

5 Friesen $R H$, Honda AT, Thieme RE. Changes in anterior fontanel pressure in preterm neonates during tracheal intubation. Anesth Analg 1987; 66: 874-8
6 Lerman J, Robinson S, Willis MM, Gregory GA. Anesthetic requirements for halothane in young children 0-1 month and 1-6 months of age. Anesthesiology 1983; 59: $421-4$.

7 LeDez KM, Lerman J. The minimum alveclar concentration (MAC) of isoflurane in preterm neonates. Anesthe. siology 1987; 67: 301-7.

8 Robinson S, Gregory GA. Fentanyl-air-oxygen anesthesia for ligation of patent ductus arteriosus in preterm infants. Anesth Analg 1981; 60: 331-4.

9 Meakin G, Shaw EA, Baker RD, Morris P. Comparison of atracurium-induced neuromuscular blockade in neonates, infants and children. Br J Anaesth 1988; 60: 171-5.

10 Arthur DS, McNicol LR. Local anaesthetic techniques in paediatric surgery. Br J Anaesth 1986, 58:760-8.

11 Coté CJ, Goldstein EA, Coté MA, Hoaglin DC, Ryan $J F$. A single-blind study of pulse oximetry in children. Anesthesiology 1988; 68: 184-8.

12 MacDonald NJ, Fitzpatrick GJ, Moore KP, Wren WS, Keenan $M$. Anaesthesia for congenital hypertrophic pyloric stenosis. A review of 350 patients. Br J Anacsth 1987; 59: 672-7.

$13 \mathrm{Diaz} J \mathrm{H}$. Tension pneumoperitoneum - pneumothorax during repair of a congenital diaphragmatic hernia. Anesth Analg 1987; 66: 577-80.

14 Yaster M, Buck JR, Dudgeon DL et al. Hemodynamic effects of primary closure of omphalocele/gastroschisis in human newborns. Anesthesiology 1988; 69: 84-8.

\section{General references}

15 Rothstein $P$. Anesthesia for surgical emergencies in the neonate. American Society of Anesthesiologists Refresher Course 1987; 15: 177-87.

16 Loftness SL, Lockhart CH. Pitfalls in the use of anesthetic agents in children. Int Anesth Clin 1985; 23: 201-26.

17 Dierdorf $S F$, Krishna $G$. Anesthetic management of neonatal surgical emergencies. Anesth Analg 1981; 60:204-15. 